\title{
Adaptive Approaches to Mobile Learning
}

This special issue of the International Journal of Interactive Mobile Technologies (iJIM) is focused on the theme of Adaptive Approaches to Mobile Learning. The significant growth of wireless technology in recent years, increasing availability of high bandwidth network infrastructures, advances in mobile technologies and the popularity of handheld devices have opened up new accessibility opportunities for education. The true potential of elearning as "anytime, anywhere" has finally begun to be realized in the form of m-learning, not only for those with disabilities or those living in remote communities, but also for those who have been attending traditional academia but could benefit from improved collaboration possibilities, situated learning opportunities and contextual learning.

Adaptive scenarios such as the use of multimedia-rich content, design of appropriate learning tasks, and use of collaborative paradigm, have potential to take mobile learning to a level where it can deliver education, at a higher quality than is presently available in a classroom environment, surpassing that by incorporating contextual problem scenarios from learners' working and social environments, thereby, enabling collaborative problemsolving in authentic environmental contexts, such as problem themes based on the real entities in the learners' immediate environment.

The articles in this special issue span across the changes in pedagogy that is needed to support adaptive and contextual learning in mobile environment and the technology developments that are needed to make it happen. With the help of real use cases and evaluative scenarios, the special issue is aimed to be archival resource for both practitioners and researchers of today and tomorrow.

The first paper in this special issue by Hansen and Bouvin focuses in contextual adaptation in mobile learning and presents the HyCon framework for mobile, contextaware, and multi-platform hypermedia. The paper contains both theoretical underpinning of the framework and multitude of practical applications that have been built using the framework. The paper provides a deep reflection of the advantages that contextual adaptation can provide and validates its applicability through evaluation of the applications developed using the framework.

Second paper is by Laroussi and Derycke, taking the adaptivity in mobile learning one step further towards realization. Authors discuss the standardization of adaptivity in mobile learning through extending the existing IMS Learner Information Package (LIP), and provide an editor to facilitate the implementation and update of the extended LIP by teachers.

Next paper in this special issue is by Tan and Kinshuk, focusing on the design principles of developing adaptive mobile learning systems. Analysing the characteristics of the mobile devices and the environment in which they operate, authors elicit five principles for designing mobile learning systems, and explain these principles through their application in the development of an adaptive mobile learning system.

The final paper in the special issue is by Eteokleous and Ktoridou, discussing very practical issues regarding integration of mobile learning in higher education sector in Cyprus. Based on the findings of deployment of mobile devices in real academic environment, authors argue that the success of mobile learning integration demands 
serious consideration towards adapting the deployment through appropriate technological and pedagogical considerations.

We would like to thank following experts who helped in the review process to ensure that the special issue serves as an archival resource for both practitioners and researchers: Mohamed Ally, Tzung-Shi Chen, Shu-Chen Cheng, Sheng-Hui Hsu, Yueh-Min Huang, Wu-Yuin Hwang, Mike T.H. Liang, Hiroaki Ogata, Rahman Samia, Harris Wang, Xiaokun Zhang.

The Guest Editors:

Prof. Kinshuk

Director School of Computing and Information Systems

Athabasca University

Canada

Prof. Yueh-Min Huang

Chair Dept. Engineering Science

National Cheng-Kung University

Taiwan

Dr. Qing Tan

School of Computing and Information Systems

Athabasca University

Canada 